\title{
AVALIAÇÃO E SELEÇÃO DE PROGÊNIES DE CUPUAÇUZEIRO (Theobroma grandiflorum), EM BELÉM, PARÁ ${ }^{1}$
}

\author{
RAFAEL MOYSÉS ALVES², MARCOS DEON VILELA DE RESENDE ${ }^{3}$, \\ BRUNA DOS SANTOS BANDEIRA4 ${ }^{4}$, THIAGO MARTINS PINHEIRO ${ }^{5}$, \\ DANIELLA CRISTINA RAIOL FARIAS ${ }^{6}$
}

RESUMO- Este trabalho teve por objetivo a seleção entre e dentro de progênies de irmãos completos de cupuaçuzeiro. Foram avaliadas 21 progênies de irmãos completos, em dois experimentos, com cinco plantas por parcela. Os experimentos foram avaliados ao nível de indivíduos, em cada safra, para os caracteres: produção de frutos, polpa e semente, bem como resistência à vassoura-de-bruxa. Para efeito de seleção, também foi considerado o grau de parentesco das matrizes. As análises foram conduzidas via metodologia de modelos lineares mistos, como delineamento em blocos incompletos, desbalanceados com tratamentos comuns. Foram estimados os parâmetros genéticos e os valores genotípicos ajustados de progênies, bem como os valores genéticos aditivos e genotípicos individuais. Os resultados demonstraram que as progênies 12; 13; 18; 20; 21 e 24 tiveram os melhores desempenhos para as variáveis de produção. Entre elas, as progênies 12; 13 e 18 foram as que mais se destacaram. Foram selecionadas três matrizes da progênie 18, duas matrizes da progênie 12 e uma matriz de cada uma das progênies 13; 20 e 21. É possível concluir que essas matrizes, juntamente com materiais selecionados em outras áreas experimentais, apresentam potencial para compor um pomar de sementes clonais, estabelecido em lote isolado de outros plantios, onde será produzida uma população melhorada de primeiro ciclo que se constituirá na nova cultivar de cupuaçuzeiro.

Termos para indexação: Progênies de irmãos completos, seleção genotípica, vassoura-de-bruxa.

\section{EVALUATION AND SELECTION OF Theobroma grandiflorum PARENTS, IN BELEM, PARÁ}

\begin{abstract}
This paper had as objective the selection among and within full sib families of Theobroma grandiflorum. A total of 21 full sibs progenies were evaluated, in two experiments, with five plants per plot. The experiments were evaluated at the individuals' level, in each crop, for the traits: production of fruits, pulp and seed and resistance to witch's broom disease. The degree of genetic relationship of the candidates was also considered. The analyses were performed through the methodology of mixed linear models, as outlined for unbalanced incomplete blocks with common treatments. The genetic parameters were obtained, as well as the genotypic and the addictive genetic values for individuals. The results showed that the progenies 12 , 13, 18, 20, 21 and 24 were the best for the production variables, with the progenies 12, 13 and 18 being the best ones. Three mother trees of the progeny 18, two of the progeny 12 and one of each one of the progenies: 13, 20 and 21, were selected. It's possible to conclude that those mother trees together with materials selected in other experimental areas will compose a clonal seed orchard, providing the new cultivar of $T$. grandiflorum. Once selected and cloned, those mother trees should compose a seed orchard, isolated from other plantations, for the production of an improved population of first cycle.
\end{abstract}

Index terms: Full sibs, genotypic selection, witch’s broom disease.

\footnotetext{
${ }^{1}$ (Trabalho 025-09). Recebido em: 07-01-2009. Aceito para publicação em: 05-08-2009. Suporte financeiro: Embrapa e CNPq.

2Doutor, Embrapa Amazônia Oriental; Belém - Pará, rafael@cpatu.embrapa.br

32Doutor, Embrapa Florestas, Colombo - Paraná, marcos.deon@gmail.com

${ }^{4}$ Engenheira Agrônoma - Universidade Federal Rural da Amazônia, Belém - Pará, bruban6@hotmail.com

${ }^{5}$ Engenheiro Agrônomo,- Universidade Federal Rural da Amazônia, Belém - Pará, thiago_mpinheiro@yahoo.com.br

${ }^{6}$ Engenheira Agrônoma - Universidade Federal Rural da Amazônia, Belém - Pará, daniellaraiolfarias@yahoo.com.br
} 


\section{INTRODUÇÃO}

O cultivo do cupuaçuzeiro, Theobroma grandiflorum (Willd. ex Spreng.) Schum., teve início no Estado do Pará, nos anos de 1970 e, posteriormente, foi expandido para toda a região amazônica. Dentre os problemas enfrentados para tornar o cupuaçuzeiro um cultivo sustentável, destaca-se a doença conhecida como vassoura-de-bruxa, causada pelo fungo Crinipellis (Moniliophthora) perniciosa, (Stahel) Singer, que ataca ramos, flores e frutos. Essa doença vem acarretando danos econômicos significativos às plantações, obrigando produtores das áreas tradicionais a abandonar seus cultivos, tornando-se um dos fatores limitantes à expansão da lavoura (Alves et al., 1998). Nos últimos anos, a redução da produtividade observada dos cultivos na região amazônica (Souza, 2007) tem como uma das causas a vassoura-de-bruxa.

A doença ataca também outras espécies do gênero, como o cacaueiro (Theobroma cacao L.) que, em casos severos, promove perdas anuais de produção de 20 a $90 \%$ (Dias \& Rezende, 2001), sendo a responsável por o Brasil ter perdido a posição de maior exportador de cacau do mundo (Mascarenhas et al., 2000).

Com a ampliação das áreas de cultivo de T. grandiflorum, essa enfermidade tornou-se cada vez mais significativa. A doença promove diferentes níveis de danos, variando de uma planta para outra, em razão, provavelmente, do controle genético e de fatores ambientais (Alves et al., 1997). A utilização de cultivares resistentes, aliada ao emprego de técnicas que minimizem a propagação de epidemias, como adubação balanceada, plantio multiespecíficos, nível de sombreamento equilibrado, podas profiláticas, uso de fungicidas eficientes, entre outros, são estratégias que, futuramente, promoverão melhor sanidade e, consequentemente, maior sustentabilidade às lavouras (Cruz et al., 2000; Cruz \& Alves, 2001).

A primeira tentativa para obter materiais resistentes à M. perniciosa teve início em 1986, com a coleta de acessos aparentemente resistentes à enfermidade, em diferentes localidades da Amazônia e, posteriormente, formação do BAG de cupuaçuzeiro de Belém (Lima \& Costa, 1991). Em 2002, a Embrapa Amazônia Oriental lançou uma cultivar policlonal, composta pelos clones Coari, Codajas, Manacapuru e Belém, que se constituiu no primeiro material de plantação do cupuaçuzeiro caracterizado pela tolerância à vassoura-de-bruxa e boa produção de frutos (Alves \& Cruz, 2003).
A estratégia de obtenção de clones visando à exploração do vigor heterótico, em que características de resistência à vassoura-debruxa sejam associadas à alta produção de frutos, continuará a ser uma linha de pesquisa promissora, pois poderá conferir elevado ganho genético por ciclo de melhoramento, dando respostas mais imediatas para a demanda de material de plantação. Entretanto, essa estratégia oferece riscos de quebra da resistência, uma vez que o produto dessa linha de pesquisa são clones, normalmente, com base genética restrita. Trata-se de materiais mais recomendados para produtores tecnificados, que terão condições de agregar, ao controle genético, caso necessário, outras práticas agronômicas, como controle fitossanitário, envolvendo aplicações de fungicidas e poda profilática das vassouras (ramos) e frutos atacados. Para tentar minimizar o problema, a seleção dos clones deverá ser efetuada a campo, para que as manifestações de resistência horizontal sejam também consideradas (Alves, 2003).

Porém, para a grande demanda, constituída pela agricultura familiar e pequenos plantadores, 0 tipo de material de plantação deve ser diferente, assim como a estratégia para obtê-lo. Somente pelo melhoramento populacional será possível oferecer sementes de cupuaçuzeiro com ampla base genética (Alves, 1999; Souza et al., 2002).

Este trabalho visa a selecionar matrizes de cupuaçuzeiro geneticamente resistentes à vassourade-bruxa, com boa produção de fruto, polpa e semente, a partir da avaliação de progênies de irmãos completos, utilizando uma nova estratégia para avaliação e seleção de matrizes. Após clonagem, essas matrizes deverão compor uma quadra (pomar de sementes), isolada de demais plantios, para a produção de uma população melhorada de primeiro ciclo, que se constituirá na próxima cultivar de cupuaçuzeiro.

\section{MATERIAL E MÉTODOS}

O experimento foi instalado em fevereiro de 1998, na Base Física da Embrapa Amazônia Orien-

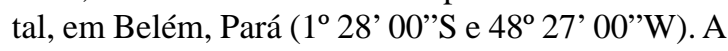
área experimental apresenta solo do tipo Latossolo Amarelo textura média, profundo, bem drenado, com baixa fertilidade e acidez elevada. O clima é do tipo Afi, com temperatura média de $25,9^{\circ} \mathrm{C}$, precipitação pluviométrica de $3.200 \mathrm{~mm}$ e umidade relativa de $85 \%$.

O material experimental constou de 21 progênies de irmãos completos (Tabela 1). Na produção dessas progênies, foram utilizados como parentais 
quatro cultivares lançadas em 2002 pela Embrapa Amazônia Oriental: 174 (Coari), 186 (Codajas), 215 (Manacapuru) e 286 (Belém), além de clones altamente produtivos, porém suscetíveis à $M$. perniciosa, como: 1074, 434, entre outros. Os materiais foram avaliados em dois experimentos, no delineamento experimental de blocos casualizados, com cinco plantas por parcela. O primeiro experimento avaliou 16 progênies, com 3 repetições, e o segundo, 13 progênies, com 2 repetições. Os dois experimentos foram conectados por 8 progênies comuns a eles e foram empregadas como testemunhas progênies de meios-irmãos dos clones 174; 286 e 618.

Foram avaliados 370 indivíduos em quatro safras anuais, perfazendo um total de 1.484 observações analisadas. O experimento foi analisado ao nível de indivíduos em cada safra, para os caracteres: produção de frutos, produção de polpa e produção de sementes. Foi avaliada, também, a intensidade de ocorrência de vassoura-de-bruxa nos ramos, verificando-se a presença ou ausência de sintomas (vassoura nos ramos) durante toda a fase experimental.

As análises foram realizadas pela metodologia de modelos lineares mistos (procedimento REML/ BLUP), como um delineamento em blocos incompletos desbalanceados com tratamentos comuns. Isso permitiu o ajuste para os gradientes ambientais de blocos e também a recuperação de informação genética interblocos incompletos, propiciando eficiente estimação de parâmetros e seleção. Os efeitos de blocos foram considerados como aleatórios, visando a propiciar a recuperação da informação genética interblocos. Dessa forma, os parâmetros genéticos foram estimados via REML, e os valores genotípicos ou médias genotípicas ajustadas de progênies, via BLUP. Da mesma forma, os valores genéticos aditivos e genotípicos individuais foram estimados pelo procedimento BLUP, por meio do software Selegen-Reml/Blup (Resende, 2002). Utilizou-se o seguinte modelo estatístico para a avaliação genética (Dias \& Resende, 2001; Resende, 2002):

$\mathrm{y}=\mathrm{Xm}+\mathrm{Zg}+\mathrm{Wp}+\mathrm{Tb}+\mathrm{Qc}+\mathrm{e}$, em que:

$y, m, g, p, b, c, e=$ vetores de dados, de efeitos de medições (fixos), de efeitos genotípicos de progênies de irmãos germanos (aleatório), de efeitos permanentes de indivíduos (aleatório), de efeitos de blocos (aleatórios), de efeitos de parcela (aleatórios) e de erros aleatórios, respectivamente.

$\mathrm{X}, \mathrm{Z}, \mathrm{W}, \mathrm{T}, \mathrm{Q}=$ matrizes de incidência para $\mathrm{m}, \mathrm{g}, \mathrm{p}, \mathrm{b}$ e c, respectivamente.

Equações de modelo misto, em que:

$$
\begin{aligned}
& {\left[\begin{array}{ccccc}
X^{\prime} X & X^{\prime} Z & X^{\prime} W & X^{\prime} T & X^{\prime} H \\
Z^{\prime} X & Z^{\prime} Z+A^{-1} \lambda_{1} & Z^{\prime} W & Z^{\prime} T & Z^{\prime} H \\
W^{\prime} X & W^{\prime} Z & W^{\prime} W+I \lambda_{2} & W^{\prime} T & W^{\prime} H \\
T^{\prime} X & T^{\prime} Z & T^{\prime} W & T^{\prime} T+I \lambda_{3} & T^{\prime} H \\
H^{\prime} X & H^{\prime} Z & H^{\prime} W & H^{\prime} T & H^{\prime} H+I \lambda_{4}
\end{array}\right]\left[\begin{array}{c}
\hat{m} \\
\hat{g} \\
\hat{p} \\
\hat{b} \\
\hat{c}
\end{array}\right]=\left[\begin{array}{c}
X^{\prime} y \\
Z^{\prime} y \\
W^{\prime} y \\
T^{\prime} y \\
H^{\prime} y
\end{array}\right]} \\
& \lambda_{1}=\frac{1-\rho}{g^{2}}=\frac{\sigma_{e}^{2}}{\sigma_{g}^{2}} ; \quad \lambda_{2}=\frac{1-\rho}{p^{2}}=\frac{\sigma_{e}^{2}}{\sigma_{p}^{2}} ; \quad \lambda_{3}=\frac{1-\rho}{b^{2}}=\frac{\sigma_{e}^{2}}{\sigma_{l}^{2}} \quad \lambda_{4}=\frac{1-\rho}{c^{2}}=\frac{\sigma_{e}^{2}}{\sigma_{c}^{2}} . \\
& g^{2}=\frac{\sigma_{g}^{2}}{\sigma_{g}^{2}+\sigma_{c}^{2}+\sigma_{p}^{2}+\sigma_{e}^{2}+\sigma_{b}^{2}} \quad \text { coeficiente de determinação dos efeitos de progênie, em } \\
& \rho=\frac{\sigma_{g}^{2}+\sigma_{c}^{2}+\sigma_{p}^{2}+\sigma_{l}^{2}}{\sigma_{g}^{2}+\sigma_{c}^{2}+\sigma_{p}^{2}+\sigma_{e}^{2}+\sigma_{b}^{2}} \quad \text { : repetibilidade individual. } \\
& p^{2}=\frac{\sigma_{p}^{2}}{\sigma_{g}^{2}+\sigma_{c}^{2}+\sigma_{p}^{2}+\sigma_{e}^{2}+\sigma_{b}^{2}} \quad \text { : coeficiente de determinação dos efeitos permanentes dentro }
\end{aligned}
$$

uma dada medição. de progènie. 


$$
\begin{gathered}
c^{2}=\frac{\sigma_{c}^{2}}{\sigma_{g}^{2}+\sigma_{c}^{2}+\sigma_{p}^{2}+\sigma_{e}^{2}+\sigma_{b}^{2}} \text { : coeficiente de determinação dos efeitos de coluna. } \\
b^{2}=\frac{\sigma_{l}^{2}}{\sigma_{g}^{2}+\sigma_{c}^{2}+\sigma_{p}^{2}+\sigma_{e}^{2}+\sigma_{b}^{2}} \quad: \text { coeficiente de determinação dos efeitos de bloco. }
\end{gathered}
$$

Estimadores iterativos dos componentes de variância por REML via algoritmo em:

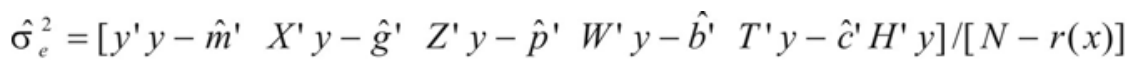

$$
\begin{aligned}
& \hat{\sigma}_{g}^{2}=\left[\hat{g}^{\prime} \hat{g}+\hat{\sigma}_{e}^{2} \operatorname{tr}\left(C^{22}\right)\right] / f \\
& \hat{\sigma}_{c}^{2}=\left[\hat{c}^{\prime} c+\hat{\sigma}_{e}^{2} \operatorname{tr} C^{55}\right] / s \\
& \hat{\sigma}_{p}^{2}=\left[\hat{p}^{\prime} \hat{p}+\hat{\sigma}_{e}^{2} \operatorname{tr} C^{33}\right] / q \\
& \hat{\sigma}_{b}^{2}=\left[\hat{b}^{\prime} \hat{b}+\hat{\sigma}_{e}^{2} \operatorname{tr} C^{44}\right] / r
\end{aligned}
$$

C22, C33, C44 e C55 advêm da inversa da matriz dos coeficientes das equações de modelo misto.

$\mathrm{N}, \mathrm{f}, \mathrm{s}, \mathrm{q}, \mathrm{r}=$ número total de dados, número de progênies, número de parcelas, número de indivíduos e número de blocos, respectivamente.

\section{RESULTADOS E DISCUSSÃO}

A seleção de matrizes de cupuaçuzeiro, normalmente, é baseada em variáveis isoladas, como: número de frutos, peso de frutos, rendimento de polpa e rendimento de sementes (Alves et al., 1997; Souza et al., 1998). Essas variáveis conduzem a números não comparáveis para efeito de seleção. Existem plantas que produzem muitos frutos, porém, com peso reduzido. Outras com frutos grandes, mas com poucos frutos por planta. Portanto, para que o processo de seleção seja eficiente, há necessidade de calcular novos parâmetros que reúnam pelo menos duas variáveis simultaneamente. Em cacaueiro, alguns índices são utilizados para as estimativas dos componentes de produção, como o Índice de Frutos (número de frutos necessários para se obter $1 \mathrm{~kg}$ de amêndoas fermentadas e secas); e o Índice de Sementes (peso médio por amêndoa obtido de 100 amêndoas fermentadas e secas). Isso porque a produção de amêndoas secas é um caráter quantitativo, portanto muito influenciado pelo ambiente (Soria, 1977).

No presente trabalho, foi utilizada uma metodologia para estimar a produção de frutos em situações nas quais, em função de furto, não é possível coletar e pesar todos os frutos produzidos por árvore, uma realidade muito comum quando as áreas experimentais ficam próximas a centros urbanos, ou quando a pesquisa é desenvolvida de forma participativa, nas áreas dos produtores (Santos et al. 2008a, 2008b). A alternativa para estimar a produção é por meio da contagem dos frutos próximos à maturação, mas ainda presos à árvore. Como a safra se estende por, aproximadamente, seis meses, período este em que é possível encontrar frutos nos diferentes estádios de maturação, há necessidade de realizar três contagens (avaliações) de frutos: no início, meio e final de safra. Como o intervalo entre as avaliações é de dois meses, fica reduzida a possibilidade de recontagem dos frutos, a qual levaria à superestimativa de produção, visto que todos os frutos contados numa avaliação já deverão ter caído na subsequente. Uma amostra de cinco frutos por planta por safra é levada ao laboratório, para a obtenção das variáveis: peso médio de frutos, peso médio de polpa e peso médio de sementes, respectivamente. As estimativas de produção média de frutos por planta e por safra, bem como a produção de polpa e semente, são então obtidas pelo produto do número de frutos produzidos por árvore, pelo peso médio dos frutos, peso médio da polpa e peso médio das sementes, respectivamente.

Essas estimativas oferecem uma base mais segura para comparações estatísticas, e a seleção torna-se mais efetiva, graças ao melhoramento simultâneo de vários componentes (Dias \& Resende, 2001). Definidas as matrizes mais produtivas, elas devem passar pelo segundo “crivo” de seleção, qual seja, a resistência à $M$. perniciosa .

No presente trabalho, a seleção foi realizada entre e dentro de progênies de irmãos completos, com base na predição BLUP dos valores genéticos, levando-se em conta o resultado de todas as variáveis estudadas. Plantas que se destacaram com alta produção, mas estavam atacadas pela doença, foram, nessa primeira seleção, descartadas. Foi levado em conta, também, o grau de parentesco das matrizes, pois a seleção visava à instalação de um pomar de sementes. 
Além do risco de endogamia, plantas geneticamente muito próximas apresentam redução na produção de frutos, em razão de problemas de incompatibilidade (Silva, 1996). Como um pomar formado somente por irmãos completos levaria à produção de sementes endogâmicas, foi estabelecido que seriam selecionadas de duas a três matrizes, no máximo, com grau de parentesco de irmãos completos. Em cacaueiro, para evitar problemas de auto e incompatibilidade, sementes de, pelo menos, cinco híbridos de cruzamentos biclonais são misturadas antes de serem entregues para os plantios comerciais (Dias \& Rezende, 2001).

Nos resultados demonstrados na Tabela 1, as progênies 12; 18; 13; 20; 21 e 24 destacaram-se quanto à produção de frutos, suplantando a primeira testemunha 174. Essas mesmas progênies foram destaque quanto à produção de polpa (Tabela 1), inferindo-se que existe uma correlação positiva entre essas duas variáveis. Análises suplementares confirmaram essa premissa, mostrando uma correlação genética positiva de 0,97 entre produção de frutos e produção de polpa.

No tocante à variável produção de sementes (Tabela 1), houve também boa consistência com os resultados anteriores, isto é, as melhores progênies quanto à produção de frutos e polpa também o são quanto à produção de sementes. Análises suplementares confirmaram também essa premissa, mostrando correlações genéticas de 0,93 entre as variáveis: produção de frutos e produção de sementes, e de 0,91 entre as variáveis: produção de polpa e produção de sementes. Almeida et al. (1994) encontraram em cacau correlação genética positiva entre número de frutos sadios por árvore e peso de amêndoas secas por árvore, concluindo que a seleção direta para número de frutos poderia maximizar a seleção para produção.

Esses resultados indicam que a seleção de materiais mais produtivos para fruto ou polpa não prejudica a seleção de materiais para produção de sementes, pois esse é hoje um nicho importante na cadeia produtiva do cupuaçuzeiro. Similarmente, no melhoramento genético do cacaueiro, a seleção também busca identificar matrizes com boa produção de frutos sadios por planta e com elevado peso das amêndoas secas por fruto (Almeida et al., 1994).

Para todas as variáveis estudadas, as progênies 12; 13 e 18 foram os destaques, abrindo a possibilidade da multiplicação dessas progênies, que poderia ser realizada em lotes isolados, com plantio em linhas alternadas dos respectivos parentais. Para evitar problemas de baixa variabilidade, após a produção das sementes nos lotes isolados, estas seriam misturadas antes de serem entregues aos produtores.
Essa estratégia não será implementada imediatamente, pois, nessa primeira fase, busca-se uma população melhorada que, além de boa produção, ofereça ampla e diversificada base genética para resistência à $M$. perniciosa. Essas três progênies, por apresentarem entre si parentesco do tipo meios-irmãos, cujo ancestral comum é o que confere os genes de resistência, não oferecem o grau de variabilidade necessário para manter a sustentabilidade dos cultivos.

Levando-se novamente em consideração a produção de frutos, a produção de polpa e a produção de sementes, além da resistência ao $M$. perniciosa e a proximidade genética, foi realizada a seleção dentro das progênies que se destacaram. $\mathrm{O}$ valor genético aditivo das 15 melhores matrizes para produção de frutos, assim como para produção de polpa, encontra-se relacionado, respectivamente, nas Tabelas 2 e 3. As duas variáveis foram capazes de identificar 11 (73\%) das 15 matrizes mais produtivas. Entre as variáveis: produção de fruto e produção de semente (Tabela 4), o nível de coincidência foi de $67 \%$, representado por 10 matrizes. E entre as três variáveis, simultaneamente, foi de $53 \%$, ou seja, oito matrizes. Verifica-se uma predominância de matrizes descendentes das progênies 12 e 18, sendo que ambas apresentam como ancestral comum o clone 186 (Codajás).

Das 15 matrizes mais promissoras em termos de produção de frutos, foi verificado que duas mostravam suscetibilidade à $M$. perniciosa (Tabela 5) e não foram consideradas para seleção. Porém, dadas as suas potencialidades de produção, vão continuar no programa de melhoramento do cupuaçuzeiro.

Foram selecionadas três matrizes da progênie 18 , duas matrizes da progênie 12 e uma matriz de cada uma das progênies: 13; 20 e 21, totalizando oito matrizes selecionadas. A comparação do comportamento dessas matrizes, em relação às variáveis estudadas, encontra-se na Tabela 6. Verifica-se que os resultados foram consistentes entre as variáveis, indicando que as matrizes poderão ser clonadas para funcionarem como progenitores no pomar de sementes. Essas sementes representarão a terceira geração, a partir das cultivares lançadas pela Embrapa Amazônia Oriental, em 2002.

Ganhos genéticos de 82,09\%, 96,23\% e $75,00 \%$ sobre o valor médio das testemunhas foram estimados para os caracteres produção de frutos, produção de polpa e produção de sementes, respectivamente (Tabela 6). As magnitudes dos ganhos genéticos demonstram o potencial dessas matrizes como progenitores da nova cultivar. 
TABELA 1 - Valores genotípicos ou médias genotípicas de progênies (irmãos completos) de cupuaçuzeiro para produção $(\mathrm{kg})$ de frutos por planta, produção $(\mathrm{kg})$ de polpa por planta e produção (kg) de sementes frescas por planta, em ensaio estabelecido em Belém, Pará, 2008.

\begin{tabular}{|c|c|c|c|c|c|}
\hline Progênie & Parentais $^{(1)}$ & $\begin{array}{l}\text { Valores genotípicos } \\
\text { para produção de } \\
\text { frutos }(\mathrm{kg}) \\
\end{array}$ & $\begin{array}{l}\text { Ordem para } \\
\text { produção de } \\
\text { frutos } \\
\end{array}$ & $\begin{array}{c}\text { Valores genotípicos } \\
\text { para produção de } \\
\text { polpa (kg) }\end{array}$ & $\begin{array}{l}\text { Valores genotípicos } \\
\text { para produção de } \\
\text { sementes }(\mathrm{kg}) \\
\end{array}$ \\
\hline 1 & $174 \times 186$ & 10,09 & 19 & 3,34 & 1,29 \\
\hline 2 & $174 \times 286$ & 11,97 & 15 & 4,04 & 1,61 \\
\hline 3 & $174 \times 513$ & 11,67 & 17 & 3,89 & 1,54 \\
\hline 4 & $174 \times 554$ & 7,20 & 24 & 2,39 & 0,93 \\
\hline 5 & $174 \times 620$ & 12,39 & 13 & 4,63 & 1,74 \\
\hline 6 & $174 \times 624$ & 12,41 & 12 & 4,18 & 1,70 \\
\hline 7 & $186 \times 554$ & 10,82 & 18 & 3,68 & 1,46 \\
\hline 8 & $554 \times 434$ & 12,47 & 11 & 3,97 & 1,88 \\
\hline 10 & $434 \times 1074$ & 10,07 & 20 & 3,81 & 1,23 \\
\hline 12 & $186 \times 434$ & 18,80 & 1 & 6,41 & 2,52 \\
\hline 13 & $186 \times 215$ & 16,63 & 3 & 6,06 & 2,53 \\
\hline 14 & $286 \times 215$ & 11,92 & 16 & 4,34 & 2,00 \\
\hline 15 & $434 \times 174$ & 13,08 & 8 & 4,50 & 1,86 \\
\hline 17 & $554 \times 624$ & 9,83 & 21 & 3,75 & 1,29 \\
\hline 18 & $186 \times 1074$ & 18,53 & 2 & 7,19 & 2,20 \\
\hline 19 & $554 \times 1074$ & 9,73 & 22 & 3,59 & 1,44 \\
\hline 20 & $186 \times 513$ & 16,37 & 4 & 5,92 & 2,36 \\
\hline 21 & $215 \times 624$ & 15,11 & 5 & 5,60 & 2,37 \\
\hline 22 & $434 \times 513$ & 8,25 & 23 & 2,81 & 1,11 \\
\hline 23 & $554 \times 620$ & 13,04 & 9 & 5,00 & 2,13 \\
\hline 24 & $286 \times 513$ & 14,56 & 6 & 5,48 & 2,13 \\
\hline 174 & PMI & 13,19 & 7 & 4,22 & 1,67 \\
\hline 286 & PMI & 12,52 & 10 & 4,40 & 1,90 \\
\hline 618 & PMI & 12,02 & 14 & 4,18 & 1,59 \\
\hline
\end{tabular}

(1)As progênies 174; 286 e 618 são meios-irmãos (PMI), usadas como testemunhas.

TABELA 2 -Valores genéticos aditivos individuais (baseados na média de 4 safras) dos 15 melhores indivíduos na população experimental de cupuaçuzeiro para o caráter produção (kg) de frutos por planta, visando à propagação sexuada, em ensaio de progênies de cupuaçuzeiro, estabelecido em Belém, Pará, 2008.

\begin{tabular}{c|c|c|c|c}
\hline Ordem & Progênie & Bloco & Planta & $\begin{array}{c}\text { Valor Genético Aditivo } \\
\text { (Produção frutos - kg) }\end{array}$ \\
\hline 1 & 12 & 1 & 2 & 26,86 \\
2 & 18 & 4 & 2 & 26,25 \\
3 & 12 & 5 & 1 & 23,25 \\
4 & 12 & 2 & 1 & 23,15 \\
5 & 13 & 2 & 2 & 22,48 \\
6 & 18 & 5 & 2 & 21,86 \\
7 & 18 & 4 & 3 & 21,82 \\
8 & 12 & 2 & 2 & 21,65 \\
9 & 12 & 1 & 3 & 21,37 \\
10 & 21 & 5 & 5 & 20,51 \\
11 & 12 & 3 & 3 & 20,43 \\
12 & 12 & 4 & 1 & 20,39 \\
13 & 20 & 4 & 1 & 20,18 \\
14 & 13 & 1 & 2 & 19,98 \\
15 & 12 & 3 & 5 & 19,90 \\
\hline
\end{tabular}


TABELA 3 - Valores genéticos aditivos individuais (baseados na média de 4 safras) dos 15 melhores indivíduos na população experimental de cupuaçuzeiro para o caráter produção (kg) de polpa por planta, visando à propagação sexuada, em ensaio de progênies de cupuaçuzeiro, estabelecido em Belém, Pará, 2008.

\begin{tabular}{c|c|c|c|c}
\hline Ordem & Progênie & Bloco & Planta & $\begin{array}{c}\text { Valor Genético Aditivo } \\
\text { (Produção polpa - kg) }\end{array}$ \\
\hline 1 & 18 & 4 & 2 & 10,01 \\
2 & 13 & 2 & 2 & 8,90 \\
3 & 18 & 4 & 3 & 8,76 \\
4 & 18 & 5 & 2 & 8,11 \\
5 & 12 & 5 & 1 & 8,09 \\
6 & 12 & 2 & 1 & 8,00 \\
7 & 12 & 1 & 2 & 7,86 \\
8 & 21 & 5 & 5 & 7,82 \\
9 & 24 & 5 & 5 & 7,56 \\
10 & 18 & 5 & 3 & 7,47 \\
11 & 18 & 5 & 1 & 7,43 \\
12 & 20 & 4 & 1 & 7,36 \\
13 & 18 & 4 & 4 & 7,30 \\
14 & 13 & 1 & 2 & 7,15 \\
15 & 12 & 1 & 3 & \\
\hline
\end{tabular}

TABELA 4 -Valores genéticos aditivos individuais (baseados na média de 4 safras) dos 15 melhores indivíduos na população experimental de cupuaçuzeiro para o caráter produção (kg) de sementes por planta, visando à propagação sexuada, em ensaio de progênies de cupuaçuzeiro, estabelecido em Belém, Pará, 2008.

\begin{tabular}{c|c|c|c|c}
\hline Ordem & Progênie & Bloco & Planta & $\begin{array}{c}\text { Valor Genético Aditivo } \\
\text { (Produção sementes - kg) }\end{array}$ \\
\hline 1 & 13 & 2 & 2 & 3,61 \\
2 & 21 & 5 & 5 & 3,47 \\
3 & 13 & 1 & 3 & 3,32 \\
4 & 13 & 1 & 2 & 3,18 \\
5 & 12 & 2 & 1 & 3,15 \\
6 & 12 & 5 & 1 & 3,13 \\
7 & 12 & 1 & 2 & 3,05 \\
8 & 12 & 4 & 1 & 2,93 \\
9 & 21 & 3 & 1 & 2,91 \\
10 & 12 & 2 & 5 & 2,90 \\
11 & 18 & 4 & 2 & 2,89 \\
12 & 21 & 5 & 1 & 2,87 \\
13 & 12 & 3 & 3 & 2,86 \\
14 & 12 & 5 & 2 & 2,80 \\
15 & 12 & 1 & 3 & 2,77 \\
\hline
\end{tabular}

TABELA 5 - Comparação entre os 15 indivíduos mais produtivos na população experimental de cupuaçuzeiro, no tocante ao caráter resistência à vassoura-de-bruxa, visando à propagação sexuada, em ensaio de progênies de cupuaçuzeiro, estabelecido em Belém, Pará, 2008.

\begin{tabular}{c|c|c|c|c}
\hline Ordem & Progênie & Bloco & Planta & Resistência à vassoura-de-bruxa \\
\hline 1 & 12 & 1 & 2 & sim \\
2 & 18 & 4 & 2 & sim \\
3 & 12 & 5 & 1 & sim \\
4 & 12 & 2 & 1 & não \\
5 & 13 & 2 & 2 & sim \\
6 & 18 & 5 & 2 & sim \\
7 & 18 & 4 & 3 & sim \\
8 & 12 & 2 & 2 & sim \\
9 & 12 & 1 & 3 & não \\
10 & 21 & 5 & 5 & sim \\
11 & 12 & 3 & 3 & sim \\
12 & 12 & 4 & 1 & sim \\
13 & 20 & 4 & 1 & sim \\
14 & 13 & 1 & 2 & sim \\
15 & 12 & 3 & 5 & \\
\hline
\end{tabular}


TABELA 6 - Seleção de oito matrizes de cupuaçuzeiro, tomando por base o ranking para os caracteres: produção de fruto, produção de polpa, produção de semente e resistência à vassoura-de-bruxa, média da população melhorada e ganho genético em relação às testemunhas, em ensaio de progênies de cupuaçuzeiro, estabelecido em Belém, Pará, 2008.

\begin{tabular}{c|c|c|c|c|c|c|c}
\hline Ordem & Progênie & Bloco & Planta & $\begin{array}{c}\text { Produção fruto } \\
(\text { Rank })\end{array}$ & $\begin{array}{c}\text { Produção polpa } \\
(\text { Rank })\end{array}$ & $\begin{array}{c}\text { Produção semente } \\
\text { (Rank) }\end{array}$ & $\begin{array}{c}\text { Resistência à } \\
\text { vassoura }\end{array}$ \\
\hline 1 & 12 & 1 & 2 & 1 & 7 & 7 & Sim \\
2 & 18 & 4 & 2 & 2 & 1 & 11 & Sim \\
3 & 12 & 5 & 1 & 3 & 5 & 6 & Sim \\
4 & 13 & 2 & 2 & 5 & 2 & 1 & Sim \\
5 & 18 & 5 & 2 & 6 & 4 & 25 & Sim \\
6 & 18 & 4 & 3 & 7 & 3 & 36 & Sim \\
7 & 21 & 5 & 5 & 10 & 8 & 18 & Sim \\
8 & 20 & 4 & 1 & 13 & 12 & 3,01 & \\
Média & - & - & - & 22,90 & 8,37 & & \\
melhorada & & & & & & & \\
Ganho & - & - & - & $82,09 \%$ & $96,23 \%$ & $75.00 \%$ & \\
genético & & & & & & & \\
\hline
\end{tabular}

\section{CONCLUSÕES}

1- A avaliação de progênies de irmãos completos de cupuaçuzeiro permitiu selecionar oito novas matrizes, com heterose para produção de frutos, polpa e sementes, mantendo, aparentemente, o mesmo grau de resistência de campo à vassoura-de-bruxa apresentada pelo parental resistente.

2- As matrizes aqui selecionadas apresentaram potencial para compor um pomar de sementes clonais e, juntamente com materiais identificados em outras áreas experimentais, darão origem à nova cultivar de cupuaçuzeiro.

\section{AGRADECIMENTOS}

Ao CNPq e à Embrapa, pelo suporte financeiro desta pesquisa. Aos empregados da Embrapa Amazônia Oriental José Raimundo Quadros Fernandes, José do S. Oliveira de Aviz, Marcos Vinícius F. da Silva, Antônio Fontel M. Pinheiro e Pedro Bezerra de Oliveira, pelos trabalhos realizados em campo.

\section{REFERÊNCIAS}

ALMEIDA, C.M.V.C.; VENCOVSKY, R.; CRUZ, C.D.; BARTLEY, B.G.D. Path analysis of yield components of cacao hybrids (Theobroma cacao L.). Brazilian Journal of Genetics, Ribeirão Preto, v. 17. p. 181-186. 1994.
ALVES, R.M. Caracterização genética de populações de cupuaçuzeiro Theobroma grandiflorum (Willd.ex.Spreng.) Schum., por marcadoresmicrossatélitesedescritoresbotânicoagronômicos. 2003. 146 f. Tese (Doutorado) Escola Superior de Agricultura "Luiz de Queiroz”, Universidade de São Paulo, Piracicaba, 2003.

ALVES, R.M. Cupuaçuzeiro (Theobroma grandiflorum Willd. ex. Spreng) Schum). In: EMBRAPA. Programa de melhoramento genético e de adaptação de espécies vegetais para a Amazônia Oriental. Belém: Centro de Pesquisa Agroflorestal da Amazônia Oriental, 1999. cap.1, p.37-48. (Documentos, 16)

ALVES, R.M.; CORRÊA, J.R.V.; RODRIGO, M. Melhoramento genético do cupuaçuzeiro (Theobroma grandiflorum) no Estado do Pará. In: SEMINÁRIO INTERNACIONAL SOBRE PIMENTA-DO-REINO E CUPUAÇU, 1., 1996, Belém. Anais... Belém: EMBRAPA- CPATU, 1997. p.127-146.

ALVES, R.M.; CRUZ, E.D. Cultivares de cupuaçuzeiro tolerantes à vassoura-de-bruxa. Belém: Embrapa Amazônia Oriental, 2003. 4p. Recomendações Técnicas. 
ALVES, R.M.; STEIN, R.L.B.; ARAÚJO, D.G. de; PIMENTEL, L. Avaliação de clones de cupuaçuzeiro quanto à resistência a vassoura-de-bruxa. Revista Brasileira de Fruticultura, Jaboticabal, v.20, n.3, p.297-306, 1998.

CRUZ, E.D.; ALVES, R.M. Avaliação de clones de cupuaçuzeiro, Theobroma grandiflorum (Willd ex Spreng) Schum, na Amazônia Oriental. In: SIMPÓSIO DE RECURSOS GENÉTICOS PARA A AMÉRICA LATINA E CARIBE, 3., Londrina. 2001. Anais. Londrina: IAPAR/Embrapa Recursos Genéticos e Biotecnologia, 2001. p.281-283.

CRUZ, E.D.; ALVES, R.M.; BENCHIMOL, R.L. Avaliação de clones de cupuaçuzeiro (Theobroma grandiflorum (Willd ex Spreng) Schumm) quanto a tolerância à vassoura-de-bruxa (Crinipellis perniciosa (Stahel) Singer). Belém: Embrapa Amazônia Oriental, 2000. 4p. (Comunicado Técnico, 28)

DIAS, L. A. dos S. ; RESENDE, M. D. V. Experimentação no Melhoramento. In: DIAS, L.A.S. (Org.). Melhoramento genético do cacaueiro. Viçosa, MG: FUNAPE, 2001. v.1, p. 439-492.

LIMA, R.R.; COSTA, J.P.C. da. Registro de introduções de plantas de cultura précolombiana coletadas na Amazônia Brasileira. Belém: EMBRAPA, CPATU, 1991. 191p. (Série Documentos, 58).

MASCARENHAS, G.C.C.; MIDLEJ, R.R.; TREVISAN, S.D.P. O cluster do cacau no sul da Bahia, Brasil. In: THEORETICAL AND APPLIED GENETICS RESEARCH CONFERENCE, 13., 2000, Kuala Lumpur. Proceedings... 2000. (Paper, 72)

RESENDE, M.D.V. de. Genética biométrica e estatística no melhoramento de plantas perenes. Brasília: Embrapa Informação Tecnológica, 2002. $975 \mathrm{p}$.
SANTOS, V.S.; PINHEIRO, T.M.; ALVES, R.M. Avaliação e seleção de progênies de cupuaçuzeiro resistentes à vassoura-de-bruxa, no município de Tomé-Açu, Pará. In: CONGRESSO BRASILEIRO DE FRUTICULTURA, 20., 2008. Vitória. Anais... 2008a. CD- ROM, DVD.

SANTOS, V.S.; PINHEIRO, T.M.; ALVES, R.M. Comportamento de progênies de Theobroma grandiflorum (cupuaçuzeiro), em Tomé-Açu, Pará. In: CONGRESSO BRASILEIRO DE FRUTICULTURA, 20., 2008. Vitória. Anais... 2008b. CD-ROM, DVD.

SILVA, R.M. Estudo do sistema reprodutivo e divergência genética em cupuaçuzeiro (Theobroma grandiflorum Willd. ex Spreng.) Schum. 1996. 151 f. Dissertação (Mestrado) Escola Superior de Agricultura "Luiz de Queiroz", Universidade de São Paulo, Piracicaba, 1996.

SORIA, J. The genetic and breeding of cacao. In: INTERNATIONAL COCOA RESEARCH CONFERENCE, 5., 1977. Ibadan. Proceedings... Ibadan: Cocoa Research Institute of Nigeria, 1977. p. 18-24.

SOUZA, A. das G. C. Boas práticas agrícolas da cultura do cupuaçuzeiro. Manaus: Embrapa Amazônia Oriental, 2007. 56 p.

SOUZA, A. das G.C.; SILVA, S.E.L.; SOUZA, N.R. Avaliação de progênies de cupuaçuzeiro (Theobroma grandiflorum (Willd. ex Spreng, Schum) em Manaus. Revista Brasileira de Fruticultura, Jaboticabal, v.20, n.3, p.307-312, 1998.

SOUZA, A. das G. C. ; RESENDE, M. D. V. ; SILVA, S. E. L. ; SOUZA, N. R. . The cupuaçuzeiro genetic improvement program at Embrapa Amazônia Ocidental. Crop Breeding And Applied Biotechnology, Londrina, v. 2, n. 3, p. 471-478, 2002. 